\title{
Estrés oxidativo y actividad antioxidante en leche de vacas con mastitis subclínica
}

\author{
Aranguren, A.J.; Flores, C.A.; Meléndez, C.E.; Márquez, Y.C.; L.Ortega, A.A. \\ Unidad de Investigación en Ciencias Funcionales "Dr. Haity Moussatché" (UNIHM), Facultad de Ciencias \\ Veterinarias, Universidad Centroccidental "Lisandro Alvarado" (UCLA), Barquisimeto 3001, Venezuela. \\ Telef.: 0251-2592409, Fax 2582404, E-mail: aleidyaranguren@ucla.edu.ve
}

\begin{abstract}
Resumen
Aranguren, A.J.; Flores, C.A.; Meléndez, C.E.; Márquez, Y.C.; L.Ortega, A.A.: Estrés oxidativo y actividad antioxidante en leche de vacas con mastitis subclínica. Rev. vet. 28: 2, 103-107, 2017. La mastitis es una patología costosa en la ganadería lechera, su mayor incidencia ocurre en el periparto. El objetivo del presente estudio fue determinar el estrés oxidativo (EO) y la actividad antioxidante (AA) en la leche de vacas con mastitis. Se evaluaron 30 vacas mestizas Holstein x Carora en los primeros 150 días de lactancia, pertenecientes a una explotación comercial del estado Lara (Venezuela) divididas en dos grupos, cada uno de 15 animales: uno control y el otro con mastitis subclínica (MS). Se les extrajo dos muestras de leche en crioviales almacenados en nitrógeno líquido y una en tubos de ensayo que se mantuvieron a $8^{\circ} \mathrm{C}$. Como indicadores de EO se cuantificaron dienos conjugados (DC) por método de Wallin y malondialdehído (MDA) por test de sustancias reaccionantes con el ácido 2-tío barbitúrico). La AA derivada de la enzima glutatión peroxidasa (GSH-Px) y del glutatión (GSH), fue evaluada por kit comerciales. Para establecer la significación estadística de los resultados se utilizó la prueba " $t$ " de Student exigiéndose un $95 \%$ de certeza $(\mathrm{p}<0,05)$. Se observó un incremento de DC en el grupo con MS (1,41 $\pm 0,12 \mathrm{nmoles} / \mathrm{mg}$ proteínas) en relación al grupo control $(0,59 \pm 0,08 ; \mathrm{p}<0,001)$, como también de MDA $(0,38 \pm 0,02$ nmoles $/ m g$ proteínas) al comparar con el valor control $(0,29$ $\pm 0,02 ; p<0,001)$. La actividad de la GSH-Px se encontró elevada en el grupo con MS $(40,75 \pm 5,09$ $\mathrm{mmoles} / \mathrm{min} / \mathrm{ml})$ al comparar con el grupo control $(27,65 \pm 2,18 ; \mathrm{p}<0,05)$. La concentración de GSH se presentó aumentada en el grupo con MS $(273,71 \pm 37,51 \mu$ moles/l) en relación al grupo control $(150,00 \pm 21,17 ; \mathrm{p}<0,05)$. Se concluye que la mastitis subclínica induce en la leche un estado de EO, acompañado con un aumento de la actividad antioxidante. Estos resultados apoyarían la suplementación controlada con antioxidantes durante el período del periparto bovino.
\end{abstract}

Palabras clave: vaca, mastitis, estrés oxidativo, leche, actividad antioxidante.

\begin{abstract}
Aranguren, A.J.; Flores, C.A.; Meléndez, C.E.; Márquez, Y.C.; López-Ortega, A.A: Oxidative stress and antioxidant capacity in milk of subclinical mastitic cows. Rev. vet. 28: 2, 103-107, 2017. Mastitis is a expensive disease for dairy farming, the highest incidence occurs in the peripartum. The objective of this study was to determine the oxidative stress (OE) and antioxidant activity (AA) in the milk of cows with mastitis. Crossbred cows Holstein $x$ Carora $(n=30)$ were evaluated in the first 150 days of lactation, belonging to a commercial exploitation of Lara (Venezuela), divided into two groups of 15 animals each: control and one with subclinical mastitis (SM). Two milk samples were collected and stored in cryovials in liquid nitrogen. As OE indicators conjugated dienes (CD) were quantified using the Wallin and malondialdehyde (MDA) method by TBARS. The AA was evaluated by enzyme glutathione peroxidase (GSHPx) and glutathione (GSH) using a commercial kit. Data were analyzed by the "t" test. CD increased in the group with SM $(1.41 \pm 0.12 \mathrm{nmol} / \mathrm{mg}$ protein) compared to the control group $(0.59 \pm 0.08$; $\mathrm{p}<0.001)$, as well as MDA $(0.38 \pm 0.02 \mathrm{nmol} / \mathrm{mg}$ protein $)$ compared to control $(0.29 \pm 0.02$, $\mathrm{p}<0.001)$ value. GSH-Px activity was elevated in the group with SM $(40.75 \pm 5.09 \mathrm{mmol} / \mathrm{min} /$ $\mathrm{mL}$ ) compared with the control group $(27.65 \pm 2.18, \mathrm{p}<0.05)$. GSH concentration was increased in the group with SM $(273.71 \pm 37.51 \mu \mathrm{mol} / \mathrm{L})$ compared to the control group $(150.00 \pm 21.17 ; \mathrm{p}<0.05)$. It is concluded that subclinical mastitis in milk induces a state of $\mathrm{OE}$, accompanied with an increase in antioxidant activity. These results support antioxidant supplementation during the peripartum period bovine.
\end{abstract}

Key words: cow, mastitis, oxidative stress, milk, antioxidant capacity. 


\section{INTRODUCCIÓN}

La mastitis, una de las patologías más costosas que enfrenta la ganadería lechera, es definida como la inflamación de la glándula mamaria (GM), independientemente de la causa, que generalmente corresponde a una infección bacteriana ${ }^{6}$. Se presenta en dos formas. En la forma clínica se observa visiblemente el proceso inflamatorio de la GM, que en casos extremos induce septicemia o endotoxemia que pueden causar la muerte del animal ${ }^{8}$.

La forma subclínica, cuya frecuencia es 15 a 40 veces mayor que la forma clínica, no presenta signos visibles de inflamación pero reduce la producción de leche y afecta negativamente su calidad, lo que ocasiona pérdidas económicas importantes al sector agropecuario, además de incrementar la transmisión de agentes patógenos que causan mastitis ${ }^{16,26}$.

La mayor incidencia de mastitis se produce en las primeras semanas del posparto (lactancia temprana) y se relaciona con la inmunosupresión que suele ocurrir en dicha etapa ${ }^{13}$. En la vaca, el periodo de transición se caracteriza por modificaciones dramáticas en el estado endocrino de las hembras bovinas gestantes, que las prepara para los eventos del parto y de la lactogénesis ${ }^{17}$.

En esta etapa las células polimorfonucleares (PMN) presentan modificaciones provocadas por la detección de los patógenos invasores y la diapédesis hacia la glándula mamaria, lo que promueve la incidencia de mastitis ${ }^{24}$. Por otra parte se ha demostrado que, durante el período señalado, la vaca lechera aumenta su demanda metabólica, lo cual genera incremento de las necesidades de oxígeno y acrecentamiento en la producción de radicales libres (RL) del tipo "especies reactivas del oxígeno" (ERO).

Cuando el nivel de RL excede a la respuesta antioxidante endógena del animal, se origina un estado de estrés oxidativo (EO) ${ }^{15}$, el cual puede aumentar la susceptibilidad de ganado lechero, especialmente en el período de transición, para una variedad de trastornos que incluyen inmunodeficiencia, enfermedades infecciosas como neumonías, enteritis y mastitis, así como también infertilidad ${ }^{24,25}$.

En las infecciones intramamarias la actividad antibacteriana del neutrófilo se realiza a través del "estallido respiratorio" con producción de ERO como radical hidroxilo y oxígeno, fundamentales en el mecanismo de muerte dependiente de $\mathrm{O}_{2}$. Además los neutrófilos producen iones superóxido, hipoclorito y $\mathrm{H}_{2} \mathrm{O}_{2}$ para eliminar a las bacterias ${ }^{20}$. Estas ERO, aún cuando son esenciales en la respuesta al agente causal de mastitis, en exceso ocasionan daño tisular ${ }^{15}$.

EL objetivo del presente estudio fue determinar el EO en leche de vacas con mastitis subclínica, mediante la cuantificación de los parámetros de EO, dienos conjugados (DC) y malondialdhído (MDA) y de la actividad antioxidante derivada del glutatión (GSH) y de la enzima glutatión peroxidasa (GSH-Px).

\section{MATERIAL Y MÉTODOS}

Localización, población, grupos. El estudio se realizó en una unidad de producción comercial, ubicada en el sector "El Callao", vía Boro del municipio Morán del estado Lara, Venezuela, localizada a $9^{\circ} 47^{\prime} 12^{\prime} \mathrm{N}$ y 69.47'34'O, con una altitud de $635 \mathrm{msnm}$, distante 63 $\mathrm{km}$ de la ciudad de Barquisimeto. El clima es semiárido templado, temperatura media anual $27^{\circ} \mathrm{C}$, variación de $19^{\circ} \mathrm{C}$ hasta $30^{\circ} \mathrm{C}$, precipitación media anual: 973,9 mm, distribuida bimodalmente: el $83,5 \%$ en los meses de abril a noviembre y $16,5 \%$ entre diciembre y marzo, características de bosque seco tropical ${ }^{12}$.

La población estuvo conformada por un total de 545 animales en producción, presentes en el plantel, con 305 vacas en ordeño. El sistema de producción fue de tipo intensivo, con ordeño mecánico, alimento concentrado, heno picado y agua ad libitum durante el día. Los criterios de inclusión fueron: vacas de raza mestizas Holstein x Carora, con 2 o más partos, que se encontraran hasta en 150 días de lactancia.

Los criterios de exclusión fueron: vacas con más de 150 días de lactancia, que presentaran alguna patología (infecciosa, parasitaria, reproductiva, nerviosa o podal), que recibieran tratamiento antibiótico o lo hayan recibido en los últimos 15 días.

Los animales incluídos se distribuyeron en: un grupo control constituido por 15 vacas que resultaron negativas a las pruebas de fondo negro y California Mastitis Test (CMT), y en un grupo con mastitis subclínica (MS) formado por 15 vacas que en la prueba de CMT arrojaron contajes celulares mayores a 200.000 células/ $\mathrm{ml}$ de leche.

Manejo de animales, toma de muestras. La manipulación de los animales para la experimentación se realizó en cumplimiento con lo establecido en la legislación de la República Bolivariana de Venezuela, tomando como normativa a seguir el Código de Bioética y Bioseguridad (tercera edición, publicado en 2008 por el Fondo Nacional de Ciencia, Tecnología e Innovación FONACIT).

A todos los animales que cumplían con los criterios de inclusión, antes del ordeño se les realizó la prueba de fondo negro, para evidenciar presencia de grumos o coágulos en la leche, que indican alteración de sus características físicas. Las vacas negativas a esta prueba se sometieron al diagnóstico para mastitis subclínica, que se realizó con un kit de Agritech-CA para la prueba CMT ${ }^{21}$; los resultados de esta prueba fueron confirmados con el recuento electrónico de células somáticas (RECS) en leche, realizada con equipo Fossomatic Minor ${ }^{\circledR}$, según las recomendaciones de la Federación Internacional de Lechería, 1984.

A los animales seleccionados se les extrajeron 3 muestras de leche del cuarto afectado con mastitis. Una de las muestras con aproximadamente $100 \mathrm{ml}$ de leche, destinada al RECS, fue trasladada en una cava con gel congelado, a una temperatura de aproximada- 

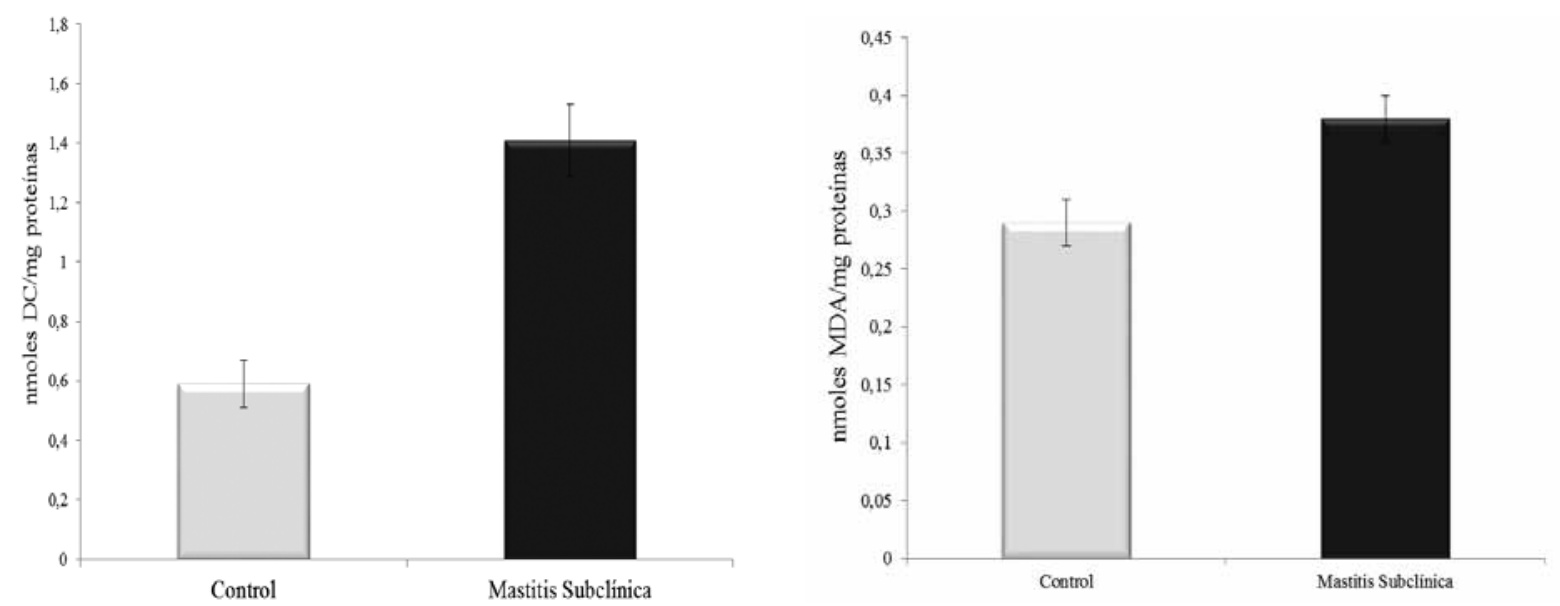

Figura 1. Parámetros de estrés oxidativo en leche de vacas con mastitis subclínica y controles. Izquierda: dienos conjugados (DC). Derecha: malondialdehído (MDA), media $\pm \mathrm{EE}, \mathrm{n}=15, * * * \mathrm{p}<0,001$.

mente $8^{\circ} \mathrm{C}$, hasta el laboratorio de Bromatología de los Alimentos, donde fue procesada. Otras dos muestras en crioviales de $2 \mathrm{ml}$ cada uno, se colocaron en una cava con hielo seco y fueron trasladadas al laboratorio de la UNIHM (UCLA), donde fueron almacenadas en nitrógeno liquido, hasta el momento de su procesamiento.

Determinación de los parámetros de EO, de la actividad antioxidante y análisis estadístico. El nivel de DC se determinó por el método descrito por Wallin ${ }^{27}$ y el MDA fue cuantificado por la prueba TBARS (test para sustancias reaccionantes con el ácido 2-tío barbitúrico) de acuerdo a la técnica de Ohkawa ${ }^{19}$.

La concentración de proteínas en leche se determinó mediante el kit comercial (Pierce, Rockford, Ill, USA), basado en la técnica descrita por Bradford ${ }^{7}$. La actividad antioxidante fue cuantificada en la leche a través de la GSH-Px y de la concentración de GSH, en ambos casos utilizando kits Calbiochem-Novabiochem Corp. (La Jolla, Ca, USA).

Los resultados obtenidos representan la media \pm error estándar (EE). El análisis estadístico fue realizado con la ayuda del paquete SPSS versión 17.0 para
Windows, mediante la prueba " $t$ " de Student, considerándose significativos los resultados con $p<0,05$.

\section{RESULTADOS}

Recuento electrónico de células somáticas (RECS) en leche. El valor promedio del RECS en leche de vacas mestizas Holstein x Carora con mastitis subclínica fue de 1.061 .200 células $/ \mathrm{ml}$ de leche, a diferencia del valor obtenido en el grupo control, de 52.571 células/ml de leche, en una explotación ubicada en el Municipio Morán, Estado Lara, Venezuela ( $n=15)$.

Parámetros de estrés oxidativo. A la izquierda de la Figura 1 se presentan las concentraciones de DC y a la derecha las concentraciones de MDA, en la leche de vacas mestizas Holstein x Carora con mastitis subclínica, y en controles. Ambos parámetros mostraron un incremento estadísticamente significativo $(p<0,001)$ en el grupo de animales con MS, en comparación con el grupo control.
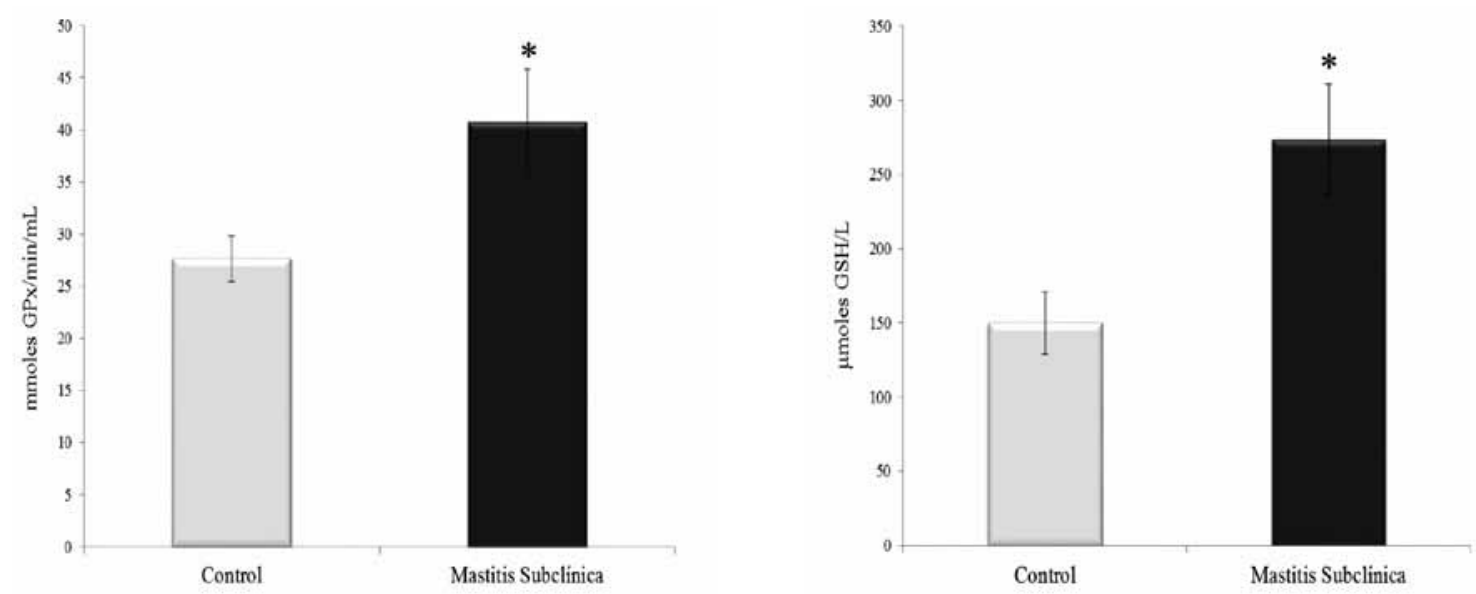

Figura 2. Actividad antioxidante en leche de vacas mestizas Holstein x Carora con mastitis subclínica y controles. Izquierda: glutatión peroxidasa (GSH-Px), derecha: glutatión (GSH), media $\pm \mathrm{EE}, \mathrm{n}=15,{ }^{*} \mathrm{p}<0,05$. 
Actividad antioxidante. Los resultados de la actividad de GSH-Px en las muestras de leche de vacas mestizas Holstein x Carora con MS y en vacas controles, se presentan en la Figura 2 (izquierda). Se observó un aumento estadísticamente significativo $(\mathrm{p}<0,05)$ en el grupo de vacas con MS, cuando se las comparó con el grupo control. En forma similar, en la concentración del GSH se apreció un aumento estadísticamente significativo $(p<0,05)$ en el grupo de vacas con MS, en relación al grupo control (Figura 2, derecha).

\section{DISCUSIÓN}

La mastitis es una patología de honda repercusión económica que enfrenta la ganadería lechera en el período del posparto. La forma subclínica es la más frecuente, caracterizándose por ausencia de signos visibles de inflamación pero con significativa reducción de la producción de leche y efecto negativo sobre la calidad de ésta. El estado de EO que presenta la vaca en el periparto hace necesario un adecuado sistema antioxidante que minimice dicho estado, evitando la posible aparición de trastornos que comprometen la salud y productividad del animal.

En el presente estudio, los niveles lácteos de DC (productos iniciales de la cadena lipoperoxidativa) y de MDA (producto final de dicha secuencia, que se origina en las membranas celulares por interacción con las ERO, derivadas del estado de EO tisular), se incrementaron en las vacas con MS, hecho indicativo del establecimiento de un estado de EO en la leche.

Este hallazgo coincide con reportes que indican que la mastitis induce un aumento de RL en la leche bovina, dando paso a un EO en la lactancia temprana ${ }^{1}$. En las condiciones subclínicas de mastitis existe una respuesta inmunológica celular a nivel de la GM, con la finalidad de frenar el crecimiento bacteriano y destruir los microorganismos causantes de la infección intramamaria ${ }^{5}$ y se incrementa el RECS en la leche siendo las células predominantes los PMN, específicamente los neutrofilos ${ }^{10}$.

La principal función de los PMN es la fagocitosis y destrucción de microorganismos, por lo cual son considerados la primera línea de defensa celular de la GM contra los gérmenes patógenos ${ }^{9}$. Su capacidad bactericida está dada por mecanismos dependientes e independientes del oxígeno.

Este último mecanismo está compuesto por enzimas presentes en los lisosomas, tales como la lisozima y la perforina, mientras que los mecanismos dependientes de oxígeno están dados por la generación de ERO. Una vez iniciado el proceso de fagocitosis, los PMN generan anión superóxido y $\mathrm{H}_{2} \mathrm{O}_{2}$, los cuales actúan como substrato para la formación de productos tóxicos ${ }^{4}$.

La elevación de los niveles de DC y de MDA en el grupo de vacas con MS correspondería al incremento en la respuesta celular, ya que si un número de bacterias relativamente pequeño logra tener acceso a la glándula mamaria, y el sistema inmune innato es competente, puede eliminar la amenaza sin signos clínicos aparentes de la enfermedad, lo que limita la producción de mediadores pro-inflamatorios ${ }^{22}$. La magnitud de la respuesta celular dependerá de la patogenicidad del germen y de la efectividad de los mecanismos de defensa de la vaca.

El EO debe ser neutralizado para que no induzca daño a las estructuras celulares, lo cual generaría disfunción tisular. En este sentido existen mecanismos antioxidantes que degradan a los RL o que impiden que actúen. Así, el radical $\mathrm{O}_{2}{ }^{\bullet}$ es transformado por la enzima superóxido dismutasa a peróxido de hidrógeno $\left(\mathrm{H}_{2} \mathrm{O}\right)$. En presencia de metales de transición (Fe o $\mathrm{Cu}$ ), mediante la reacción de Fenton, el $\mathrm{H}_{\mathrm{O}}$ puede dar origen al radical hidroxil $\left({ }^{\circ} \mathrm{OH}\right)$ un $\mathrm{RL}$ de gran poder deletéreo.

Sin embargo, este $\mathrm{H}_{2} \mathrm{O}_{2}$ es detoxicado por las enzimas catalasa y GSH-Px, esta última utilizando al GSH que se transforma a disulfuro de glutation, que posteriormente, bajo la acción de la glutatión reductasa, regenera las dos moléculas de GSH ${ }^{11}$.

Este tripétido ( $\gamma$-glutamilcisteinilglicina) sintetizado en el citosol de las células, con propiedades reductoras y nucleófilas, cumple diversas funciones, entre ellas la homeostasis redox, lo cual reduce el daño oxidativo intracelular con un papel importante en las bacterias como factor protector contra el EO generado en el huésped, la resistencia bacteriana a la acción del $\mathrm{H}_{2} \mathrm{O}_{2}$ dependería de la acumulación de GSH, que puede activar en las células el sistema GSH-glutatión peroxidasa / glutatión reductasa ${ }^{14}$.

En este estudio los resultados de la actividad antioxidante láctea derivada de la enzima GSH-Px, tuvieron una significativa elevación $(\mathrm{p}<0,05)$ en las vacas con MS (Figura 2, izquierda). Este hallazgo es coincidente con lo reportado para vacas con MS, en cuanto a un aumento de la GSH-Px en la leche, el cual se debería principalmente a la hidrólisis del complejo enzimacaseína con liberación de la GSH-Px y asímismo, a un mecanismo antioxidante de las bacterias infectantes ${ }^{3}$.

Por su parte la concentración láctea de GSH se elevó significativamente $(\mathrm{p}<0,05)$ en las vacas con MS (Figura 2, derecha). Este compuesto tiólico contribuye a la capacidad antioxidante de las células debido a que es el mayor regulador no enzimático de la homeoestasis redox intracelular. Es sustrato de la reacción enzimática de la GSH-Px y en el postparto temprano en vacas sanas, se ha determinado una disminución de su concentración en las células sanguíneas y cambios en la actividad de la tioredoxina reductasa ${ }^{23}$.

Esta enzima y otras proteínas ricas en residuos de cisteína, tienen la propiedad de reponer los niveles de GSH y por esta razón son consideradas antioxidantes celulares, con diferentes funciones durante la síntesis del ADN nuclear, como también de defensa ante el EO y en la señalización redox, lo que conlleva a regulación de la homeostasis celular ${ }^{18}$. Se afirma que la suplementación controlada de vitaminas y elementos trazas en el 
período de transición bovina, minimizaría los efectos deletéreos del EO con disminución de la incidencia de las enfermedades del post parto ${ }^{2}$.

Se concluye que la mastitis subclínica induce en la leche un estado de EO, acompañado con un aumento de la actividad antioxidante. Estos resultados apoyarían la suplementación controlada con antioxidantes durante el período del periparto bovino.

Agradecimientos. Los autores agradecen al Consejo de Desarrollo Científico, Humanístico y Tecnológico de la Universidad Centroccidental "Lisandro Alvarado" (UCLA, Venezuela), por el financiamiento otorgado a este estudio (Proyecto 036-VE-2009).

\section{REFERENCIAS}

1. Abd-Ellah MR. 2013. Role of free radicals and antioxidants in mastitis. $J$ Adv Vet Res 3: 1-7.

2. Abuelo A, Hernández J, Benedito JL, Castillo C. 2015. The importance of the oxidative status of dairy cattle in the periparturient period: revisiting antioxidant supplementation. J Anim Physiol Anim Nutric 99: 1003-1016.

3. Andrei S. Matei S, Fit N, Cernea C, Ciupe S, Bogdan S, Groza IS. 2011. Glutathione peroxidase activity and its relationship with somatic cell count, number of colony forming units and protein content in subclinical mastitis cows milk. Romanian Biotechnol Lett 16: 6209-6217.

4. Babior BM. 1984. The respiratory burst of phagocytes. $J$ Clin Invest 73: 599-601.

5. Ballou MA. 2012. Growth and development symposium: Inflammation. Role in the etiology and pathophysiology of clinical mastitis in dairy cows. J Anim Sci 90: 1466-1478.

6. Bazan R, Cervantes E, Salas G, Segura-Correa JC. 2009. Prevalencia de mastitis subclínica en cabras lecheras en Michoacán, México. Rev Cient, FCV-LUZ 19: 334338.

7. Bradford MM. 1976. A rapid and sensitive method for the cuantitation of microgram quantities of protein utilizing the principle of protein-dye binding. Anal Biochem 72: 248-254.

8. Bradley AJ. 2002. Bovine mastitis: an evolving disease. Vet $J$ 163: 1-13.

9. Burvenich C, Monfardini E, Mehrzad J, Capuco AV, Paape MJ. 2004. Role of neutrophil polymorphonuclear leukocytes during bovine coliform mastitis: physiology or pathology? Verh K Acad Geneeskd Belg 66: 97-150.

10. Craven N, Williams MR. 1985. Defenses of the bovine mammary gland against infection and prospects for their enhancements. Vet Immunol Immunopathol 10: 71-127.

11. Dröge W. 2002. Free radicals in the physiological control of cell function. Physiol Rev 82: 47-95.

12. Ewel JJ, Madriz A, Tosi JA. 1976. Zonas de vida de Venezuela. En: Memoria explicativa sobre el mapa ecológico (MAC y FNIA editores), 2da ed., Ed. Sucre, Caracas, Venezuela, p. 76-88.
13. Goff JP, Kimura K. 2001. Factors affecting the health and disease resistance of the transition cow. In: Dairy cattle nutrition workshop Penn State University. On line: http://dasweb.psu.edu/pdf/goffpaper2.pdf.

14. Li Y, Hugenholtz J, Abee T, Molenaar D. 2003. Glutathione protects Lactococcus lactis against oxidative stress. Appl Env Microbiol 69: 5739-5745.

15. Lykkesfeldt J, Svendsen O. 2007. Oxidants and antioxidants in disease: oxidative stress in farm animals. Vet $J$ 173: 502-511.

16. Mweu MM, Nielsen SS, Halasa T, Toft N. 2012. Annual incidence, prevalence and transmission characteristics of Streptococcus agalactiae in Danish dairy herds. Prev Vet Med 106: 244-250.

17. National Research Council. 2001. The nutrient requirement of dairy cattle, Seventh revised edition, National Academy Press, Washington DC, p.381.

18. Navarro M, Castro W, González S, Abad MJ, Taylor P. 2013. Synthesis and anticancer activity of Gold (I)-chloroquine complexes. J Mex Chem Soc 57: 220-229.

19. Ohkawa H, Ohihi N, Yagi K. 1978. Assay for lipid peroxides in animal tissues by thiobarbituric acid reaction. Anal Biochem 95: 351-358.

20. Rinaldi M, Moroni P, Paape MJ, Bannerman D. 2007. Evaluation of assays for the measurement of bovine neutrophil ROS. Vet Immunol Immunopathol 115: 107-125.

21. Schalm OW, Noorlander DO. 1957. Experiments and observations leading to development of the California mastitis test. J Am Vet Med Assoc 130: 199-204.

22. Schuster DE, Lee EK, Kehrli ME. 1996. Bacterial growth, inflammatory cytokine production, and neutrophil recruitment during coliform mastitis in cows within ten days after calving, compared with cows at midlactation. Am J Vet Res 57: 1569-1575.

23. Sordillo LM, O'Boyle N, Gandy JC, Corl CM, Hamilton E. 2007. Shifts in Thioredoxin reductase activity and oxidant status in mononuclear cells obtained from transition dairy cattle. J Dairy Sci 90: 1186-1192.

24. Sordillo LM, Aitken SL. 2009. Impact of oxidative stress on the health and immune function of dairy cattle, Vet Immunol Immunopathol 128: 104-109.

25. Spears JR, Weiss WP. 2008. Role of antioxidants and trace elements in health and immunity of transition dairy cows. Vet $J$ 176: 70-76.

26. Tesfaye GY, Regassa FG, Kelay B. 2010. Milk yield and associated economic losses in quarters with subclinical mastitis due to Staphylococcus aureus in Ethiopian crossbred dairy cows. Trop Anim Health Prod 42: 925-931.

27. Wallin B, Rosengren B, Shertzer H, Camejo G. 1993. Lipoprotein oxidation and measurement of thiobarbituric acid reacting substances formation in a single microtiter plate: Its use for evaluation of antioxidants. Analyt Biochem 208: 10-15. 